Article

\title{
Human-Like Walking with Heel Off and Toe Support for Biped Robot
}

\author{
Yixiang Liu ${ }^{1,2,3}$, Xizhe Zang ${ }^{1, *}$, Shuai Heng ${ }^{1}$, Zhenkun Lin ${ }^{1}$ and Jie Zhao ${ }^{1}$ \\ 1 State Key Laboratory of Robotics and System, Harbin Institute of Technology, Harbin 150080, China; \\ liuyixiang@163.com (Y.L.); heng13514479054@163.com (S.H.); 15B308008@hit.edu.cn (Z.L.); \\ jzhao@hit.edu.cn (J.Z.) \\ 2 Legs + Walking Lab, Shirley Ryan AbilityLab (Formerly the Rehabilitation Institute of Chicago), Chicago, \\ IL 60611, USA \\ 3 Department of Physical Medicine and Rehabilitation, Northwestern University, Chicago, IL 60611, USA \\ * Correspondence: zangxizhe@hit.edu.cn; Tel.: +86-451-8641-3382
}

Academic Editors: Toshio Fukuda, Fei Chen and Qing Shi

Received: 31 March 2017; Accepted: 5 May 2017; Published: 18 May 2017

\begin{abstract}
The under-actuated foot rotation that the heel of the stance leg lifts off the ground and the body rotates around the stance toe is an important feature in human walking. However, it is absent in the realized walking gait for the majority of biped robots because of the difficulty and complexity in the control it brings about. In this paper, a hybrid control approach aiming to integrate the main characteristics of human walking into a simulated seven-link biped robot is presented and then verified with simulations. The bipedal robotic gait includes a fully actuated single support phase with the stance heel supporting the body, an under-actuated single support phase, with the stance toe supporting the body, and an instantaneous double support phase when the two legs exchange their roles. The walking controller combines virtual force control and foot placement control, which are applied to the stance leg and the swing leg, respectively. The virtual force control assumes that there is a virtual force which can generate the desired torso motion on the center of mass of the torso link, and then the virtual force is applied through the real torques on each actuated joint of the stance leg to create the same effect that the virtual force would have created. The foot placement control uses a path tracking controller to follow the predefined trajectory of the swing foot when walking forward. The trajectories of the torso and the swing foot are generated based on the cart-cable model. Co-simulations in Adams and MATLAB show that the desired gait is achieved with a biped robot under the action of the proposed method.
\end{abstract}

Keywords: biped robot; human-like walking; foot rotation; virtual force control; foot placement control

\section{Introduction}

Humans are the most important inspiration for research on biped robots because of their resemblance. The unique neural and morphological mechanisms enable humans to show very versatile and efficient locomotion. Consequently, realizing human-like walking on biped robots has been an attractive research field for decades. Up to now, various advanced biped robots have been developed, from childlike robots NAO [1,2] and iCub [3,4] to human-sized robots Asimo [5,6], HRP [7-9], and Atlas $[10,11]$. For these biped robots, the most popular control approach is based on the Zero Moment Point (ZMP) theory, i.e. the contact point of the foot with the ground where the total of horizontal inertia and gravity forces equals zero is strictly within the support polygon [12,13]. Following the ZMP criterion ensures that these biped robots walk stably and robustly on a variety of terrains. However, in order to increase the stability of walking, the stance foot of biped robots is always flat on the ground, making the gait unnatural and energy-inefficient. 
Actually, closer investigation on human walking reveals that, by the end of the single support phase, the heel of the stance foot rises from the ground and rotates around the toe, with the stance toe touching the ground and supporting the body [14,15]. Even from the gait of persons with lower limb disabilities wearing prostheses can foot rotation be observed [16]. The so-called "heel off and toe support" yields a higher foot clearance, a higher walking speed, and less energy consumption [17-19]. Taking the human as a mechanical locomotion system, it will be under-actuated when foot rotation occurs because there is no actuation between the stance toe and the ground [20]. Although dealing with the changes in dynamic characteristics is seemingly effortless for humans, it is much more difficult for biped robots. Therefore, it is of great important to study human-like walking of biped robots with under-actuated foot rotation.

In order to attain this goal, some researchers have adopted the ZMP-based method [21-23]. The desired walking pattern that includes foot rotation was generated by solving an inverse kinematics problem with a predefined ZMP and foot trajectories and achieved under servo controllers such as preview control. Chevallereau et al. [24] proposed a path-following control strategy to handle more human-like walking. The control strategy was defined in such a way that only the kinematic evolution of the state of the robot was controlled, but not its temporal evolution, allowing simultaneous control of the ZMP and the joint positions. Since the ZMP could be directly regulated, stable bipedal walking that includes prescribed foot rotation was realized. Sinnet and Zhao et al. [25-27] used a human-inspired methodology to translate human locomotion to a biped robot. By analyzing human locomotion data, the human-inspired outputs of the robot were defined based on the human outputs and the representation function. Then, a controller was designed for the biped robot, enabling it to mimic human kinematics behaviors by tracking the functions of the kinematics.

Although bipedal robotic walking with under-actuated foot rotation has been realized in the above research, the methods are usually very complex. Thus, a different and simpler approach is proposed in this paper. The walking control scheme adopts a hybrid controller that combines the ideas of virtual force control and foot placement control. The virtual force control, which is applied on the stance leg, assumes that there is a virtual force on the center of mass of the torso that can generate the desired torso motion including foot rotation, and the real torques are exerted on each actuated joint of the stance leg to create the same effect that the virtual force would have created. The foot placement control, which is applied on the swing leg, uses a path-tracking controller to follow the predefined curve of the swing foot to step forward. Using this hybrid controller, the sophisticated control task of dynamic walking is disintegrated into two simpler ones, making it much easier to achieve.

The rest of this paper is organized as follows. Section 2 presents the proposed hybrid control approach. As the most prevalent reference for biped robots, the human walking pattern is analyzed first. On this basis, a seven-link biped robot model and human-like robotic gait are introduced. Then the method of how to realize human-like walking on the biped robot is introduced in detail. To verify the method, co-simulations are carried out in Adams and MATLAB, and these simulation results are shown in Section 3. Finally, some conclusions and discussion are provided in Section 4.

\section{Methods}

\subsection{Human Walking Pattern Analysis}

In order to reproduce anthropomorphic walking on a biped robot, it is important to study and understand the walking pattern of humans. Human walking is the repetition of a basic movement, namely, the step. Generally, a normal step can be roughly divided into two successive phases according to the foot contact with the ground: the double support phase (or the stance phase), when both feet are on the ground, and the single support phase (or the swing phase), when only one foot is in contact with the ground [28]. Figure 1 shows a single gait cycle, from when one foot strikes the ground with its heel to when the other foot strikes the ground. In the initial portion of the single support phase, the sole of the stance foot is flat on the ground, and the center of pressure locates within the stance 
heel. For the rest of the single support phase, the heel of the stance foot lifts off the ground and rotates around the toe [29]. The center of the pressure moves forward from the stance heel to the stance toe before the heel of the swing foot contacts the ground. The foot rotation around the toe in the single support phase is named "heel off and toe support", which is a significant feature of human walking and has an important effect on walking performance.

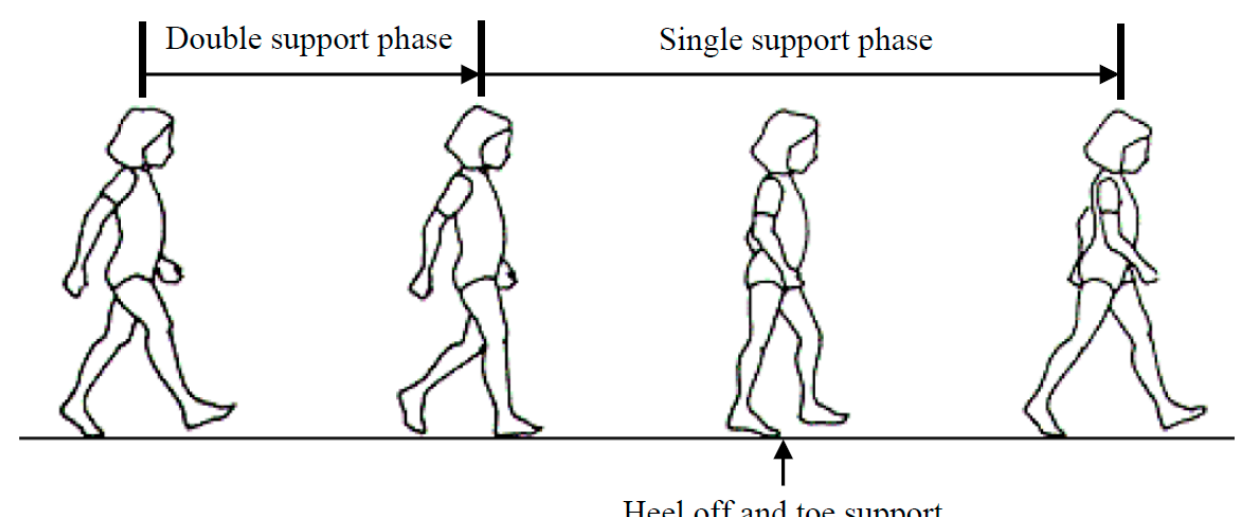

Figure 1. A single gait cycle of the human walking pattern. In the single support phase, the heel of the stance foot lifts off the ground and rotates around the toe, which is named "heel off and toe support".

\subsection{The Biped Robot Model}

The model considered in this paper is a planar seven-link biped robot of which the movement is constrained in the sagittal plane. The biped robot has two three-segmented legs connected by the right and left hip joints at the torso. Each leg is composed of a thigh, shank, and foot that are interconnected by the knee joint and the ankle joint. Each joint is regarded as an independently actuated ideal or theoretically frictionless revolute joint with only one degree of freedom (DOF). Therefore, the biped robot has a total of six internal DOFs, namely, one in each hip joint, one in each knee joint, and one in each ankle joint.

Figure 2 illustrates the biped robot model. The right and left legs are supposed to be identical and exchange their roles between steps. Thus, the properties of the two legs are pairwise equal. $l_{i}$ and $m_{i}$ represent the length and mass of link $i$, respectively. The distance between the local center of mass $(\mathrm{CoM})$ and the lower end of link $i$ is indicated by $\eta_{i} l_{i}$ where $0<\eta_{i}<1$. Having six actuated joints, the biped robot dynamics is driven by six joint driving torques, i.e., $\tau=\left[\tau_{1}, \tau_{2}, \tau_{3}, \tau_{4}, \tau_{5}, \tau_{6}\right]^{\mathrm{T}}$. At any time during a step, the configuration of the biped robot can be defined by a set of generalized coordinates, namely, $q=\left[\theta_{1}, \theta_{2}, \theta_{3}, \theta_{4}, \theta_{5}, \theta_{6}, \theta_{7}\right]^{\mathrm{T}}$. The base coordinate frame is attached to the base of the robot, i.e., the tiptoe of the stance foot. According to the kinematics theory of robot, $\theta_{i}(i=1,2,3,4,5,6,7)$ indicates the amount of rotation around the $z$-axis needed to align the axis of link $i-1$ with the axis of link $i$. Specifically, $\theta_{1}$ represents the angle around the $z$-axis from the $x$-axis of the base frame to the axis of the stance foot of the biped robot, and $\theta_{2}$ to $\theta_{7}$ represent the angles between each two adjacent links from the stance foot to the swing foot. Positive rotation follows the right hand rule. In this case, the direction of counterclockwise rotation about the $z$-axis is assumed positive. 


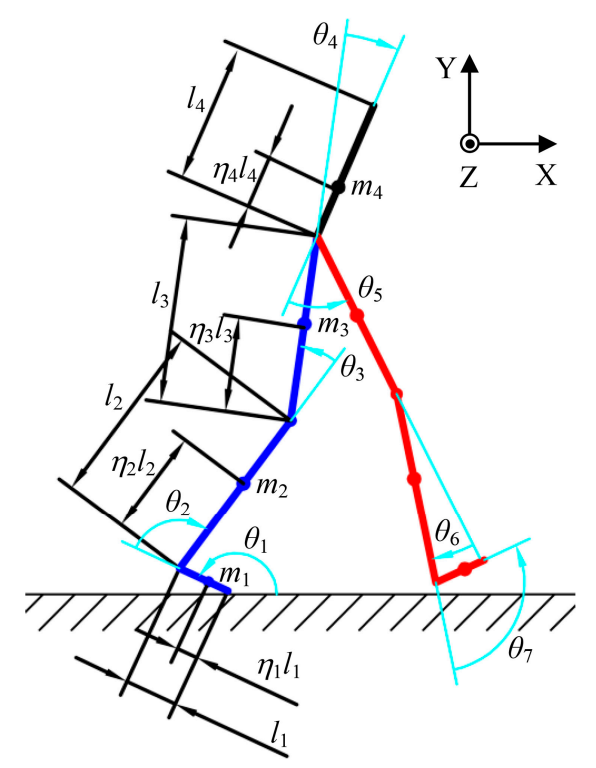

Figure 2. The seven-link biped robot model with associated geometric and inertial properties. The corresponding links on each leg are geometrically and inertially identical.

\subsection{The Bipedal Robotic Gait}

When the contact point between the foot and ground changes during walking, the biped robot translates into the next walking phase that has quite different dynamical characteristics, resulting in many difficulties to the control of the biped robot. This paper is focused on the realization of the so-called "heel off and toe support" on a biped robot. Therefore, the bipedal robotic gait is simplified from the human walking pattern to some extent. First, the double support phase is assumed to be instantaneous. Second, when the swing foot strikes the ground, the sole is parallel to the ground. The desired robotic walking motion can then be regarded as including three phases: a heel support phase, a toe support phase, and an instantaneous double support phase (or impact phase). Figure 3 shows the bipedal robotic walking pattern with state transition events. Each phase is introduced as follows.

(1) Heel support phase (HSP). This phase starts when the toe of the swing leg lifts off the ground. The whole foot of the stance leg is assumed to remain flat and motionless on the ground without slipping. At the same time, the swing leg swings in the forward direction. In this phase, the biped robot is fully actuated because it has the same numbers of degrees of freedom (DOF) and actuators.

(2) Toe support phase (TSP). This phase starts when the heel of the stance leg lifts off the ground. In this phase, the stance foot rotates around its toe, which is virtually pivoted to the ground, and the swing leg continues to swing forward. The biped robot is under-actuated because there is no actuation between the stance foot and the ground.

(3) Impact Phase (IP). Leg roles exchange takes place in this phase. After the swing foot strikes the ground, the swing leg becomes the new stance leg, while the original stance leg rises from the ground and becomes the swing leg. 


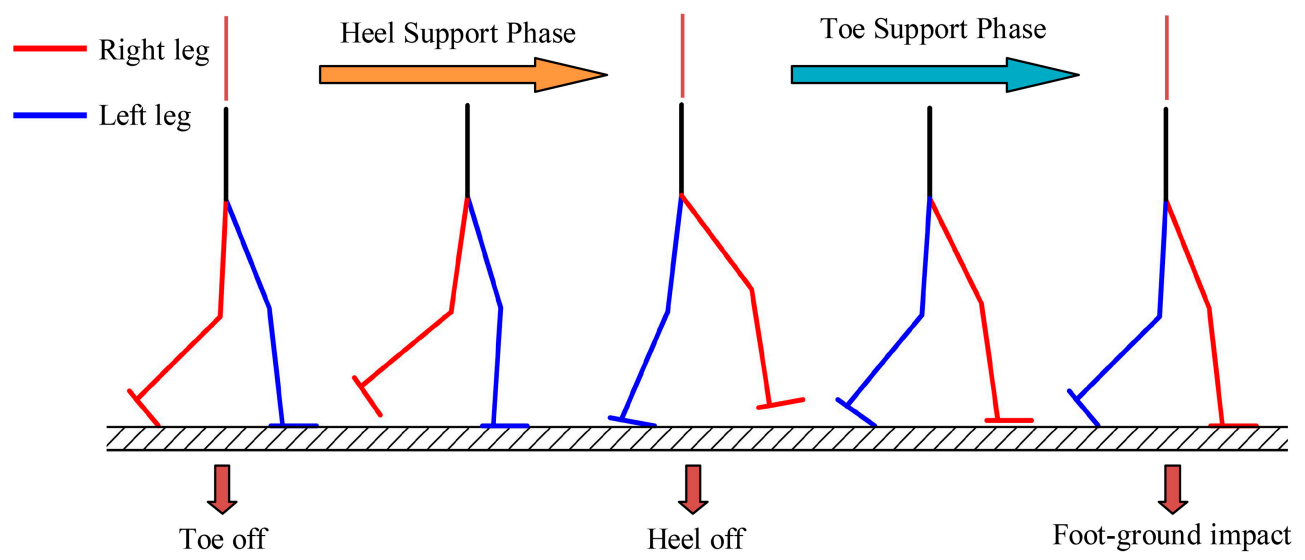

Figure 3. The bipedal robotic walking pattern, which consists of a heel support phase, a toe support phase, and an impact phase. The state transition events are toe off, heel off, and foot-ground impact, respectively.

\subsection{The Hybrid Control Scheme}

The angle between the stance foot and the ground is determined by the dynamics of the biped robot and cannot be controlled directly. Thus, the greatest difficulty in the control of biped robots is how to integrate the under-actuated toe support phase into the motion. Considering that the high dimensional walking task can be viewed as a collection of several decoupled tasks of lower dimensionality, the control of the biped robot is decoupled into two tasks, i.e., the control of the torso to track the desired motion, and the control of the swing leg to follow the predefined foot placement point. Therefore, a hybrid control scheme is proposed in this paper. The control scheme combines the ideas of virtual force control and foot placement control, which are applied on the stance leg and the swing leg, respectively. The working principle of the hybrid control scheme as illustrated in Figure 4 is detailed below.

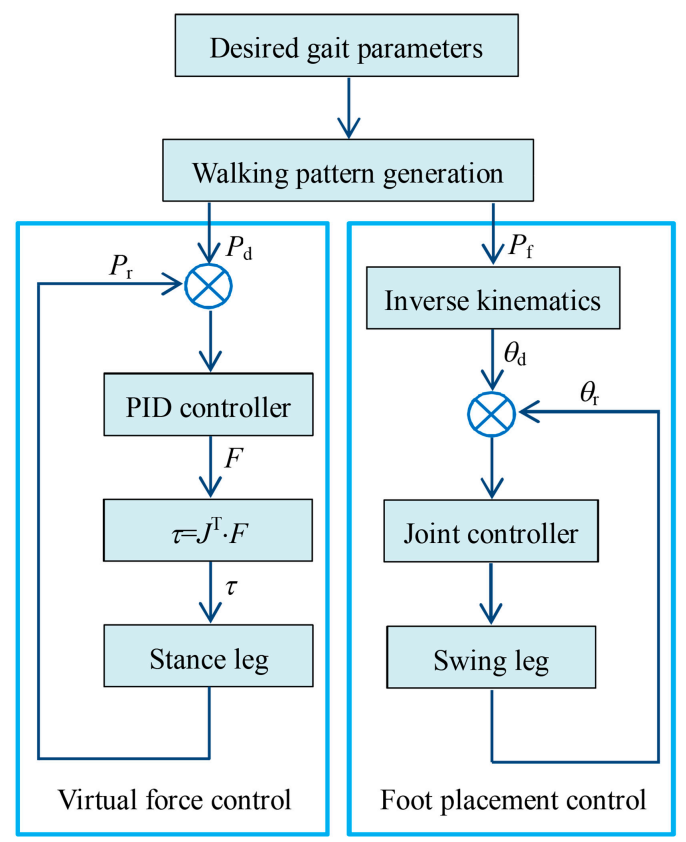

Figure 4. The hybrid walking control scheme. The virtual force control is applied on the stance leg, while the foot placement control is applied on the swing leg.

The main idea of virtual force control is quite straightforward. Taking the CoM of the torso as the end-effector, its motion in the sagittal plane is predefined by the walking pattern generator according 
to the given gait parameters. In fact, the position and posture of the torso are determined by the motion of the previous joints, i.e., from the toe to the hip of the stance leg. However, it is assumed that a virtual force is directly acted on the center of mass of the torso link, which leads to the desired torso motion. Based on the dynamics theory, the real torques on the previous joints can be derived, which create the same effect that the virtual force would have created had they existed [30].

Suppose that the origin of the global reference frame $\left(X_{0}, Y_{0}, Z_{0}\right)$ is set at the tiptoe of the stance foot. The kinematical equations of the biped robot can be easily analyzed by a geometrical method. The position vector of the local CoM of the torso indicated by $P=\left[P_{x}, P_{y}, \theta_{z}\right]^{\mathrm{T}}$ can be described as

$$
P=\left[\begin{array}{c}
P_{x} \\
P_{y} \\
\theta_{z}
\end{array}\right]=\left[\begin{array}{c}
l_{1} c_{1}+l_{2} c_{1+2}+l_{3} c_{1+2+3}+\eta_{4} l_{4} c_{1+2+3+4} \\
l_{1} s_{1}+l_{2} s_{1+2}+l_{3} s_{1+2+3}+\eta_{4} l_{4} s_{1+2+3+4} \\
\theta_{1}+\theta_{2}+\theta_{3}+\theta_{4}
\end{array}\right]
$$

where $P_{x}$ represents the position in the $x$-direction, $P_{y}$ represents the position in the $y$-direction, $\theta_{z}$ represents the posture around the $z$-axis, and, for simplicity, $c_{i}=\cos \theta_{i}, s_{i}=\sin \theta_{i}, c_{i+j}=\cos \left(\theta_{i}+\theta_{j}\right)$, and $s_{i+j}=\sin \left(\theta_{i}+\theta_{j}\right)$.

The Jacobian matrix can be obtained from the partial differentiation of the above equation:

$$
J=\left[\begin{array}{cccc}
-l_{1} s_{1}-l_{2} s_{1+2}-l_{3} s_{1+2+3}-\eta_{4} l_{4} s_{1+2+3+4} & -l_{2} s_{1+2}-l_{3} s_{1+2+3}-\eta_{4} l_{4} s_{1+2+3+4} & -l_{3} s_{1+2+3}-\eta_{4} l_{4} s_{1+2+3+4} & -\eta_{4} l_{4} s_{1+2+3+4} \\
l_{1} c_{1}+l_{2} c_{1+2}+l_{3} c_{1+2+3}+\eta_{4} l_{4} c_{1+2+3+4} & l_{2} c_{1+2}+l_{3} c_{1+2+3}+\eta_{4} l_{4} c_{1+2+3+4} & l_{3} c_{1+2+3}+\eta_{4} l_{4} c_{1+2+3+4} & \eta_{4} l_{4} c_{1+2+3+4} \\
1 & 1 & 1 & 1
\end{array}\right]
$$

Use $F=\left[F_{x}, F_{y}, T_{z}\right]^{\mathrm{T}}$ to indicate the virtual force acting on the torso, with the three components representing the force in the $x$-direction, the force in the $y$-direction, and the torque around the $z$-axis respectively. Then, the real actuator torques $\tau=\left[\tau_{0}, \tau_{1}, \tau_{2}, \tau_{3}\right]^{\mathrm{T}}$ can be calculated using the Jacobian matrix, which maps the force on the end-effector to the joint torques:

$$
\tau=J^{T} \cdot F
$$

It should be noted that the torque $\tau_{0}$ acting on the stance toe actually equals zero since the stance toe is unactuated. Therefore, the three components of the virtual force are subject to the following constraint:

$$
\tau_{1}=\left(-l_{1} s_{1}-l_{2} s_{1+2}-l_{3} s_{1+2+3}-\eta_{4} l_{4} s_{1+2+3+4}\right) F_{x}+\left(l_{1} c_{1}+l_{2} c_{1+2}+l_{3} c_{1+2+3}+\eta_{4} l_{4} c_{1+2+3+4}\right) F_{y}+T_{z}=0
$$

which means that, once any two of these three components are determined, the third one can be solved from this equation.

In this paper, the virtual force and torque $F_{y}$ and $T_{z}$ are first specified by applying two PID controllers on the torso to track the desired position in the $y$-direction and the desired posture around the $z$-axis. $F_{y}$ and $T_{z}$ can be obtained from the summation of the output of the PID controller and gravity term, written as

$$
\begin{aligned}
& F_{y}=K_{P, y}\left(P_{y, d}-P_{y, r}\right)+K_{I, y} \int_{0}^{t}\left(P_{y, d}-P_{y, r}\right) d t+K_{D, y} \frac{d\left(P_{y, d}-P_{y, r}\right)}{d t}+m_{4} g \\
& T_{z}=K_{P, z}\left(P_{z, d}-P_{z, r}\right)+K_{I, z} \int_{0}^{t}\left(P_{z, d}-P_{z, r}\right) d t+K_{D, z} \frac{d\left(P_{z, d}-P_{z, r}\right)}{d t}
\end{aligned}
$$

where $P_{y, d}$ and $P_{z, d}$ are desired positions of the torso, and $P_{y, r}$ and $P_{z, r}$ are real positions of the torso.

Combining Equations (4) and (5), the virtual force $F_{x}$ can be derived. Plugging $F_{x}, F_{y}$, and $T_{z}$ back into Equation (3), the joint torques can be calculated.

The foot placement control aims to output the appropriate step location of the biped robot to obtain the desired walking gait [31]. For the swing leg, given the walking parameters such as step length, step height, and cycle time, the trajectory of the swing foot is generated. Once the trajectories of the torso and 
swing foot are both already known, the inverse kinematics can be resolved to calculate the joint angles of the swing leg. Then, position controllers are applied on the joints to track the desired trajectories.

\section{Results}

\subsection{Walking Pattern Generation}

The role of the walking pattern generator is to output the desired trajectories of the CoM of the torso and the swing foot. In this paper, the cart-cable model shown in Figure 5 is adopted, with the cart representing the CoM of the robot, and the foot of the table representing the stance foot of the robot $[32,33]$. It is assumed that the mass of the biped robot is lumped with the CoM of the robot, and the CoM is kept at a constant height. Based on the model, the relationship between the trajectories of the CoM and the reference ZMP can be described as [34]

$$
\ddot{x}_{\mathrm{COM}}=\frac{g}{z_{\mathrm{COM}}}\left(x_{\mathrm{COM}}-x_{\mathrm{ZMP}}\right)
$$

where $x_{\mathrm{COM}}$ is the horizontal position of the $\mathrm{CoM}, x_{\mathrm{ZMP}}$ is the horizontal position of the $\mathrm{ZMP}, z_{\mathrm{COM}}$ is the height of the CoM, and $g$ is the gravity acceleration.

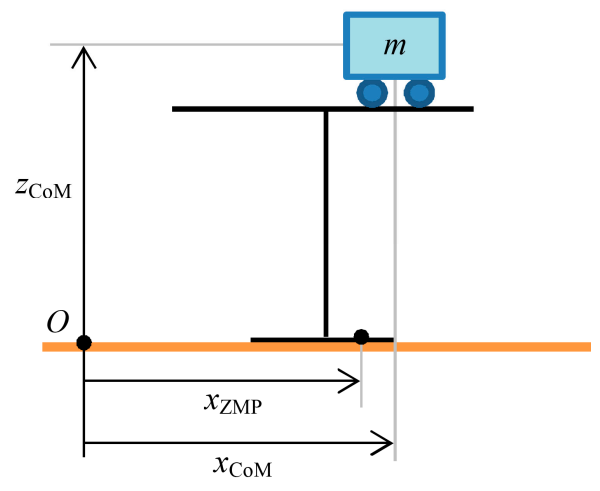

Figure 5. The cart-table model with the cart representing the center of mass (CoM) of the biped robot, and the foot of the table representing the stance foot of the robot.

The reference ZMP trajectory of the biped robot needs to be predefined according to the walking phases described previously. In the heel support phase (from time $T_{k}$ to $T_{k}+T_{h s}$ ), the ZMP moves from the heel to the toe of the stance foot. In the toe support phase (from time $T_{k}+T_{h s}$ to $T_{k+1}$ ), the ZMP is kept constant at the tiptoe. In the impact phase, the ZMP transfers from the tiptoe of the stance foot to the heel of the swing foot. Figure 6 shows the generated reference ZMP trajectory in the horizontal plane. Using Equation (6), the corresponding CoM trajectory is deduced. The trajectory of the CoM of the torso link can then be obtained by adding an offset to the reference CoM trajectory.

The trajectory of the swing foot is planned using polynomial interpolation that satisfies certain constraints in a complete walking cycle. There are four constraints on the curve, including two positional constraints and two constraints on the gradient. Thus, a polynomial of degree four is adopted to describe the trajectory. The trajectory consists of two sections related to the heel support phase and the toe support phase. The coefficients are obtained respecting the continuity of position and velocity. Moreover, in order to preserve a smooth landing of the swing foot, the velocity before the impact moment is considered to be zero. 


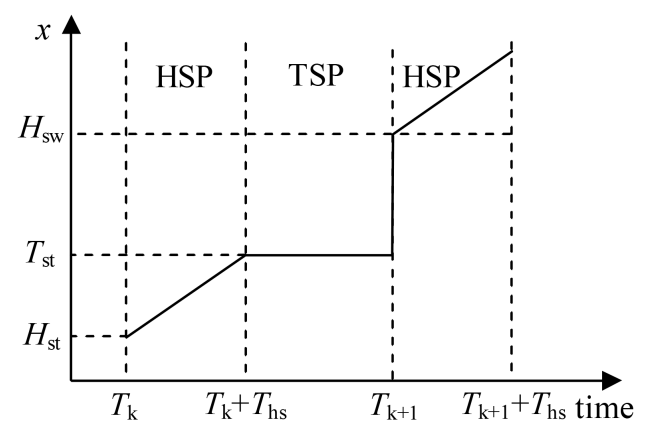

Figure 6. The reference Zero Moment Point (ZMP) trajectory of the biped robot. In the heel support phase, the ZMP moves from the heel to the toe of the stance foot. In the toe support phase, the ZMP is kept constant at the tiptoe. In the impact phase, the ZMP transfers from the tiptoe of the stance foot to the heel of the swing foot.

\subsection{Simulation}

To verify the effectiveness of the previously proposed control scheme, co-simulations are carried out in Adams and MATLAB. Adams is the most famous and widely used multibody dynamics software, and MATLAB has powerful functions in computing, programming, and control system designing. Co-simulations are able to utilize the advantages of both programs by combining the virtual mechanical system together with the complex control system. The virtual mechanical model of the biped robot is built in Adams, and the control scheme is programmed using the Simulink toolkit in MATLAB. With the Adams/Control plugin, information can be exchanged between Adams and MATLAB, in other words, outputting joint torques to the virtual model in Adams, and feeding joint motion information back to the control system in MATLAB.

The main physical parameters of the biped robot model are shown in Table 1 . The lengths of the links from the torso to the foot are $0.4,0.37,0.36$ and $0.21 \mathrm{~m}$, respectively. Their corresponding masses are $3,1.8,1.5$ and $0.3 \mathrm{~kg}$ respectively. The planned duration of the heel support phase is $0.4 \mathrm{~s}$, and the toe support phase is $0.3 \mathrm{~s}$. In the implementation of the hybrid control scheme, the main work is to tune the parameters of the controllers by trial and error. If the parameters are selected appropriately, the desired walking pattern can be realized. Figure 7 shows the screenshots of the realized bipedal robotic walking in one cycle. Figure 8 presents the joint angles of the biped robot during walking. These angles have the same meanings as defined in Figure 2. From these two figures, it is clear to see that from the start point to $0.4 \mathrm{~s}$, the sole of the stance foot is flat on the ground when the biped robot steps forward. After $0.4 \mathrm{~s}$, the angle between the ground and the stance foot sole indicated by $\theta_{1}$ begins to decrease, which means the stance heel lifts off the ground and rotates around the tiptoe. Thus, both the heel support phase and the toe support phase are realized, demonstrating that the proposed hybrid walking controller is effective.

Table 1. The main physical parameters of the biped robot model.

\begin{tabular}{ccc}
\hline Link & Length $(\mathbf{m})$ & Mass $(\mathbf{k g})$ \\
\hline Torso & 0.4 & 3 \\
Thigh & 0.37 & 1.8 \\
Shank & 0.36 & 1.5 \\
Foot & 0.21 & 0.3 \\
\hline
\end{tabular}




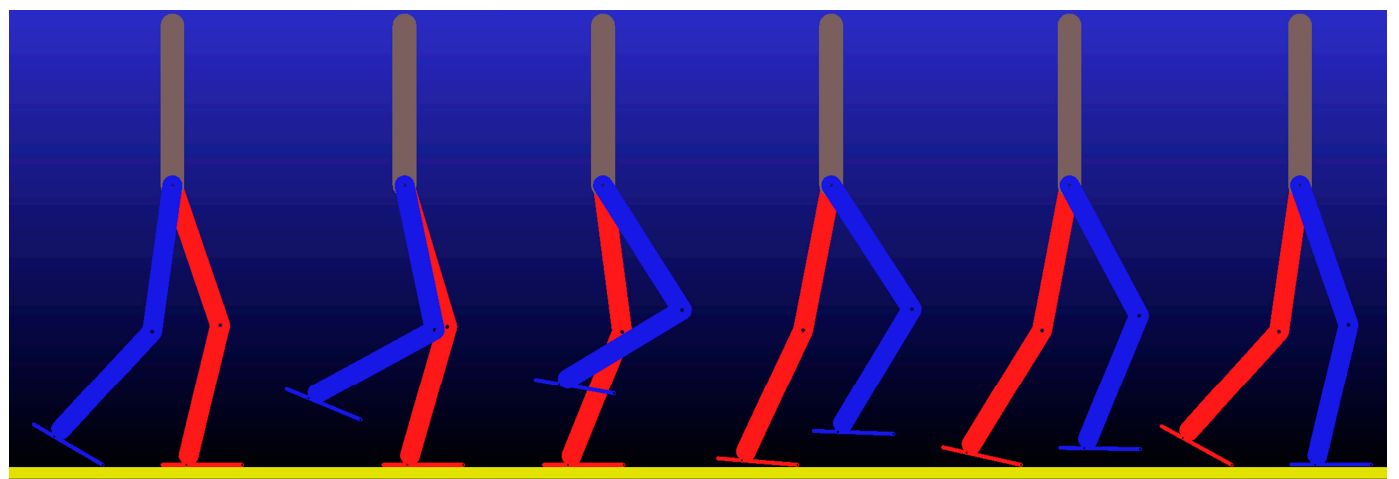

Figure 7. Snapshots of the simulated bipedal robotic walking gait in one cycle.

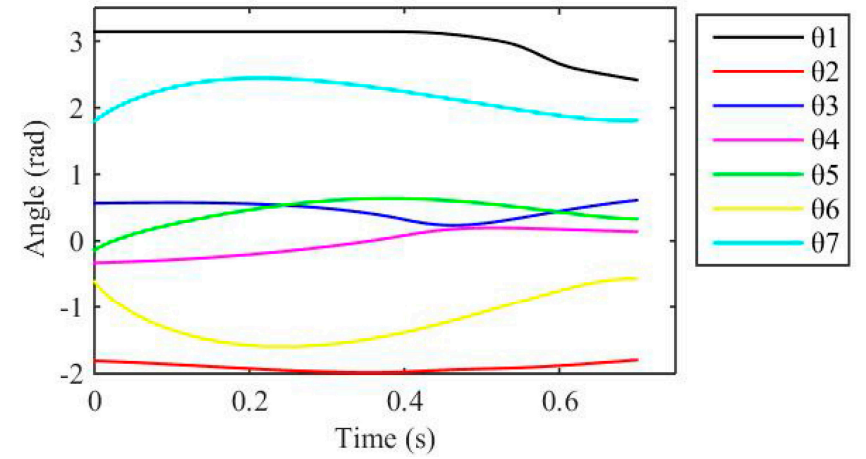

Figure 8. Joint angles of the biped robot during simulated walking. These angles have the same definitions as those in Figure 2.

\section{Conclusions and Discussion}

This paper introduces a solution to the problem of the realization of human-like bipedal robotic walking with under-actuated foot rotation. The studied biped robot model is a planar seven-like biped robot with feet. The desired gait includes three successive phases, i.e., a fully actuated phase where the heel supports the body, an under-actuated phase where the toe supports the body, and an instantaneous double support phase where the foot-ground impact takes place and the two legs exchange their roles. To achieve this gait, a hybrid walking controller is proposed by combining virtual force control and foot placement control, which are applied on the stance leg and the swing leg, respectively. The controller decouples the high-dimensional dynamic walking into two simpler tasks of lower dimensionality. Therefore, compared with the current methods, fewer control efforts and computation are required to realize human-like walking on a biped robot. The validity of the proposed approach is verified by co-simulations in Adams and MATLAB.

However, there are still limitations in this paper. The double support phase, different from the human walking pattern, is assumed to be instantaneous. In the future, we will add the finite-time double support phase to the bipedal robotic walking gait. We will also study the implementation of the hybrid control approach on a physical biped robot prototype.

Acknowledgments: The work reported in this paper was supported by the National Natural Science Foundation of China (grant no. 51675116). The first author was funded by the China Scholarship Council (grant no. 201606120094).

Author Contributions: Yixiang Liu and Xizhe Zang co-organized the work and wrote the manuscript draft. Shuai Heng and Zhenkun Lin performed the simulations. Jie Zhao supervised the research and commented on the manuscript draft.

Conflicts of Interest: The authors declare no conflict of interest. 


\section{References}

1. Nugroho, S.; Prihatmanto, A.; Rohman, A. Design and implementation of kinematics model and trajectory planning for NAO humanoid robot in a tic-tac-toe board game. In Proceedings of the 2014 IEEE 4th International Conference on System Engineering and Technology, Bandung, Indonesia, 24-25 November 2014; pp. 1-7.

2. Ghassemi, P.; Masouleh, M.; Kalhor, A. Push recovery for NAO humanoid robot. In Proceeding of the 2nd RSI/ISM International Conference on Robotics and Mechatronics, Tehran, Iran, 15-17 October 2014; pp. 35-40.

3. Tsagarakis, N.; Metta, G.; Sandini, G.; Vernon, D.; Beira, R.; Becchi, F.; Righetti, L.; Santos-Victor, J.; Ijspeert, A.; Carrozza, M.; et al. iCub: The design and realization of an open humanoid platform for cognitive and neuroscience research. Adv. Robot. 2007, 21, 1151-1175. [CrossRef]

4. Metta, G.; Natale, L.; Nori, F.; Sandini, G.; Vernon, D.; Fadiga, L.; Hofsten, C.; Rosander, K.; Lopes, M.; Santos-Victor, J.; et al. The iCub humanoid robot: An open-systems platform for research in cognitive development. Neural Netw. 2010, 23, 1125-1134. [CrossRef] [PubMed]

5. Sakagami, Y.; Watanabe, R.; Aoyama, C.; Matsunaga, S.; Higaki, N.; Fujimura, K. The intelligent asimo: System overview and integration. In Proceedings of the 2002 IEEE/RSJ International Conference on Intelligent Robots and Systems, Lausanne, Switzerland, 30 September-4 October 2002; pp. 2478-2483.

6. Kanazawa, M.; Nozawa, S.; Kakiuchi, Y.; Kanemoto, Y.; Kuroda, M.; Okada, K.; Inaba, M.; Yoshiike, T. Robust vertical ladder climbing and transitioning between ladder and catwalk for humanoid robots. In Proceedings of the 2015 IEEE/RSJ International Conference on Intelligent Robots and Systems, Hamburg, Germany, 28 September-2 October 2015; pp. 2202-2209.

7. Kaneko, K.; Harada, K.; Kanehiro, F.; Miyamori, G.; Akachi, K. Humanoid robot HRP-3. In Proceedings of the 2008 IEEE/RSJ International Conference on Intelligent Robots and Systems, Nice, France, 22-26 September 2008; pp. 2471-2478.

8. Kaneko, K.; Kanehiro, F.; Morisawa, M.; Tsuji, T.; Miura, K.; Nakaoka, S.; Kajita, S.; Yokoi, K. Hardware improvement of cybernetic human HRP-4C for entertainment use. In Proceedings of the 2011 IEEE/RSJ International Conference on Intelligent Robots and Systems, San Francisco, CA, USA, 25-30 September 2011; pp. 4392-4399.

9. Kaneko, K.; Kanehiro, F.; Morisawa, M.; Akachi, K.; Miyamori, G.; Hayashi, A.; Kanehira, N. Humanoid robot HRP-4-Humanoid robotics platform with lightweight and slim body. In Proceedings of the 2011 IEEE/RSJ International Conference on Intelligent Robots and Systems, San Francisco, CA, USA, 25-30 September 2011; pp. 4400-4407.

10. Feng, S.; Xinjilefu, X; Atkeson, C.; Kim, J. Optimization based controller design and implementation for the atlas robot in the DARPA robotics challenge finals. In Proceedings of the 2015 IEEE-RAS International Conference on Humanoid Robots, Seoul, Korea, 3-5 November 2015; pp. 1028-1035.

11. Chong, Z.; Hung, R.; Lee, K.; Wang, W.; Ng, T.; Newman, W. Autonomous wall cutting with an Atlas humanoid robot. In Proceedings of the 2015 IEEE International Conference on Technologies for Practical Robot Applications, Woburn, MA, USA, 11-12 May 2015; pp. 1-6.

12. Vukobratovic, M. Zero-moment point-Thirty-five years of its life. Int. J. Hum. Robot. 2001, 1, $157-173$. [CrossRef]

13. Vukobratović, M.; Borovac, B.; Potkonjak, V. ZMP: A review of some basic misunderstandings. Int. J. Hum. Robot. 2006, 3, 153-175. [CrossRef]

14. Adamczyk, P.G.; Collins, S.H.; Kuo, A.D. The advantages of a rolling foot in human walking. J. Exp. Biol. 2006, 209, 3953-3963. [CrossRef] [PubMed]

15. Choi, J.H.; Grizzle, J.W. Planar bipedal walking with foot rotation. In Proceedings of the 2005 American Control Conference, Portland, OR, USA, 8-10 June 2005; pp. 4909-4916.

16. Eilenberg, M.F.; Geyer, H.; Herr, H. Control of a powered ankle-foot prosthesis based on a neuromuscular model. IEEE Trans. Neural Syst. Rehabil. Eng. 2010, 18, 164-173. [CrossRef] [PubMed]

17. Tlalolini, D.; Chevallereau, C.; Aoustin, Y. Comparison of different gaits with rotation of the feet for a planar biped. Robot. Auton. Syst. 2009, 57, 371-383. [CrossRef]

18. Kouchaki, E.; Sadigh, M.J. Effect of toe-joint bending on biped gait performance. In Proceedings of the 2010 IEEE International Conference on Robotics and Biomimetics, Tianjin, China, 14-18 December 2010; pp. 697-702. 
19. Mahdokht, E.; Khadiv, M.; Moosavian, S.A.A. Effects of toe-off and heel-off motions on gait performance of biped robots. In Proceedings of the 3rd RSI International Conference on Robotics and Mechatronics, Tehran, Iran, 7-9 October 2015; pp. 007-012.

20. Tlalolini, D.; Chevallereau, C.; Aoustin, Y. Human-like walking: Optimal motion of a bipedal robot with toe-rotation motion. IEEE/ASME Trans. Mechatron. 2011, 16, 310-320. [CrossRef]

21. Sellaouti, R.; Stasse, O.; Kajita, S.; Yokoi, K.; Kheddar, A. Faster and smoother walking of humanoid HRP-2 with passive toe joints. In Proceedings of the 2006 IEEE/RSJ International Conference on Intelligent Robots and Systems, Beijing, China, 9-15 October 2006; pp. 4909-4914.

22. Kajita, S.; Kaneko, K.; Morisawa, M.; Nakaoka, S.; Hirukawa, H. ZMP-based biped running enhanced by toe springs. In Proceedings of the 2009 IEEE International Conference on Robotics and Automation, Roma, Italy, 10-14 April 2007; pp. 3963-3969.

23. Miura, K.; Morisawa, M.; Kanehiro, F.; Kajita, S.; Kaneko, K.; Yokoi, K. Human-like walking with toe supporting for humanoids. In Proceedings of the 2011 IEEE/RSJ International Conference on Intelligent Robots and Systems, San Francisco, CA, USA, 25-30 September 2011; pp. 4428-4435.

24. Chevallereau, C.; Djoudi, D.; Grizzle, J.W. Stable bipedal walking with foot rotation through direct regulation of the zero moment point. IEEE Trans. Robot. 2008, 24, 390-401. [CrossRef]

25. Sinnet, R.W.; Powell, M.J.; Shah, R.P.; Ames, A.D. A Human-inspired hybrid control approach to bipedal robotic walking. In Proceedings of the 18th World Congress/The International Federation of Automatic Control, Milano, Italy, 28 August-2 September 2011; pp. 6904-6910.

26. Zhao, H.; Ma, W.; Zeagler, M.B. Human-inspired multi-contact locomotion with AMBER2. In Proceedings of the 2014 ACM/IEEE International Conference on Cyber-Physical Systems, Berlin, Germany, 14-17 April 2014; pp. 199-210.

27. Zhao, H.; Hereid, A.; Ma, W.; Ames, A.D. Multi-contact bipedal robotic locomotion. Robotica 2017, 35, 1072-1106. [CrossRef]

28. Torricelli, D.; Gonzalez, J.; Weckx, M.; Jiménez-Fabián, R.; Vanderborght, B.; Sartori, M.; Dosen, S.; Farina, D.; Lefeber, D.; Pons, J.L. Human-like compliant locomotion: State of the art of robotic implementations. Bioinspir. Biomim. 2016, 11, 051002. [CrossRef] [PubMed]

29. Ames, A.D.; Vasudevan, R.; Bajcsy, R. Human-data based cost of bipedal robotic walking. In Proceedings of the 14th International Conference on Hybrid Systems: Computation and Control, Vienna, Austria, 12-14 April 2011; pp. 153-162.

30. Pratt, J.; Dilworth, P.; Pratt, G. Virtual model control of a bipedal walking robot. In Proceedings of the 1997 IEEE International Conference on Robotics and Automation, Albuquerque, NM, USA, 25 April 1997; pp. 193-198.

31. Li, Z.; Vanderborght, B.; Tsagarakis, N.G.; Caldwell, D.G. Human-like Walking with straightened knees, toe-off and heel-strike for the humanoid robot iCub. In Proceedings of the 2010 UKACC International Conference on Control, Coventry, UK, 7-10 September 2010.

32. Chen, X.; Zhou, Y.; Huang, Q.; Yu, Z.; Ma, G.; Meng, L.; Fu, C. Bipedal walking with toe-off, heel-strike and compliance with external disturbances. In Proceedings of the 2014 14th IEEE-RAS International Conference on Humanoid Robots, Madrid, Spain, 18-20 November 2014; pp. 506-511.

33. Kajita, S.; Kanehiro, F.; Kaneko, K.; Fujiwara, K.; Harada, K.; Yokoi, K.; Hirukawa, H. Biped walking pattern generation by using preview control of zero-moment point. In Proceedings of the 2003 IEEE International Conference on Robotics and Automation, Taipei, Taiwan, 14-19 September 2003; pp. 1620-1626.

34. Kajita, S.; Kanehiro, F.; Kaneko, K.; Yokoi, K.; Hirukawa, H. The 3D linear inverted pendulum model: A simple modeling for a biped walking pattern generation. In Proceedings of the IEEE/RSJ International Conference on Intelligent Robots and Systems, Maui, HI, USA, 29 October-3 November 2001; pp. $239-246$.

(C) 2017 by the authors. Licensee MDPI, Basel, Switzerland. This article is an open access article distributed under the terms and conditions of the Creative Commons Attribution (CC BY) license (http:// creativecommons.org/licenses/by/4.0/). 